\title{
RAUH-KÜHNE, Cornelia, RUCK, Michael, Regionale Eliten zwischen Diktatur und Demokratie. Baden und Württemberg 1930-1952
}

Hervé Joly

\section{(2) OpenEdition}

Édition électronique

URL : http://journals.openedition.org/ifha/1967

DOI : $10.4000 /$ ifha. 1967

ISSN : 2198-8943

\section{Éditeur}

IFRA - Institut franco-allemand (sciences historiques et sociales)

Référence électronique

Hervé Joly, «RAUH-KÜHNE, Cornelia, RUCK, Michael, Regionale Eliten zwischen Diktatur und Demokratie. Baden und Württemberg 1930-1952 », Revue de l'IFHA [En ligne], Date de recension, mis en ligne le 01 janvier 1995, consulté le 22 septembre 2020. URL : http://journals.openedition.org/ifha/1967 ; DOI : https://doi.org/10.4000/ifha.1967

Ce document a été généré automatiquement le 22 septembre 2020.

(C)IFHA 


\section{RAUH-KÜHNE, Cornelia, RUCK, Michael, Regionale Eliten zwischen Diktatur und Demokratie. Baden und Württemberg 1930-1952}

Hervé Joly

1 Cet ouvrage collectif est la première publication d'une nouvelle collection qui s'inscrit dans le prolongement de deux projets de recherches financés par la fondation Volkswagen. Le premier, »Résistance contre le national-socialisme dans le Sud-Ouest de l'Allemagne entre 1933 et 1945« s'intéresse à l'histoire de deux groupes sociaux, les fonctionnaires pour la partie dirigée à l'Université de Mannheim par K. Schönhoven et H. Weber, les patrons pour la partie dirigée à l'Université de Tübingen par D. Langewiesche. Le second, qui s'est achevé en 1991, portait sur le »Land de Bade sous occupation française entre 1945 et 1952«, sous la direction de H. Haumann et H.A. Winkler de l'Université de Fribourg. Le présent livre est le fruit d'une coopération entre ces différentes équipes qui vise à dépasser les barrières chronologiques de 1933 et de 1945 pour examiner l'importance des évolutions à long terme par rapport à ce que les périodes du nazisme et de l'après-guerre peuvent avoir de spécifique. La référence aux élites dans le titre ne se retrouve pas forcément dans les populations étudiées, à moins d'en adopter une définition très extensive: nombreuses sont les évocations de petits fonctionnaires ou d'artisans. Il s'agit plutôt d'une histoire régionale de deux champs sociaux, ceux de l'administration et de l'économie.

2 Le découpage en trois parties montre que l'objectif transversal n'a pas été complètement concrétisé. Dans la première partie qui porte sur la fonction publique régionale sous le nazisme, quatre chapitres ne dépassent pas les limites de la période en étudiant notamment les relations entre responsables du parti et l'administration ou la répression politique dont sont victimes certains fonctionnaires. Seule une contribution de M. RUCK correspond aux ambitions affichées. Il s'agit d'une étude très fine de l'évolution de la population des Landräte (équivalents lointains des sous-préfets 
français) de la Bade et du Wurtemberg. L'auteur montre, en mêlant données statistiques et exemples biographiques, la relative continuité, dans des régions où les fonctionnaires étaient peu engagés politiquement sous Weimar, après 1933. Rares sont les exclusions ouvertement dictées par des considérations politiques. Il est vrai que beaucoup des Landräte en fonction ont fait partie de la masse des ralliés du parti nazi du printemps 1933. Ceux qui sont écartés sont souvent reclassés dans d'autres fonctions moins sensibles. Le nouveau pouvoir utilise surtout la technique des mises à la retraite anticipée qui permet un certain renouvellement générationnel. Mais les nouveaux venus sont presque tous des fonctionnaires dont la carrière était déjà engagée avant 1933. L'interprétation de M. RUCK selon laquelle cette »autoaffirmation corporatiste a permis de préserver une capacité de résistance relativement forte« (p. 58) resterait cependant à confirmer. Les données manquent pour apprécier la portée de leur adhésion au NSDAP. Le seul Landrat qui rompt ensuite avec le régime est paradoxalement un de ceux qui, par son engagement actif dans les mouvements nationalistes sous Weimar, pouvait en paraître le plus proche. L'auteur montre en revanche bien pourquoi l'apparente discontinuité personnelle de l'après-guerre doit être relativisée: de nombreux fonctionnaires en poste en 1945 approchaient de l'âge de la retraite, ceux écartés par les autorités d'occupation bénéficient ensuite de reclassement et surtout la grande majorité de leurs successeurs jusqu'au début des années soixante-dix appartiennent à des générations formées et recrutées sous le nazisme.

3 La seconde partie sur "l'économie de l'Allemagne du Sud-Ouest et la politique économique nazie« ne déborde également guère de la période. Une contribution de $\mathrm{R}$. PETER met ainsi l'accent sur les désavantages que vaut la situation frontalière à l'industrie de la Bade: elle bénéficie peu des commandes d'armement, ses ouvriers sont beaucoup plus appelés au front et elle reçoit proportionnellement le plus faible contingent de travailleurs forcés étrangers de l'ensemble du Reich. Il est dommage que l'auteur n'évoque pas les conséquences de ces intéressantes observations dans l'économie de l'après-guerre. Le fait que l'industrie régionale ait été contrainte de s'orienter plutôt dans les biens d'équipement, la sous-traitance et les produits intermédiaires a probablement dû favoriser sa reconversion ultérieure. Seule une contribution collective qui analyse trois trajectoires de patrons du Wurtemberg qui représentent chacun trois orientations positive, médiane et négative sur l'échelle d'une plus ou moins grande proximité avec le régime, a le mérite d'en évoquer les conséquences dans l'après-guerre. P. BRÄUTIGAM, A. SCHUSTER et A. WELCK montrent bien comment ces différences de comportement ne se sont guère retrouvées dans les jugements des chambres d'épuration (Spruchkammern), qui se sont contentées de classer le plus favorable au régime dans la catégorie inoffensive des »suiveurs" (Mitläufer) et les deux autres dans celle des »déchargés« (Entlasteten).

La troisième partie consacrée à la "Dénazification et au renouveau démocratique dans la zone d'occupation française « comprend notamment des études détaillées sur l'épuration du patronat du Wurtemberg-Hohenzollern (C. RAUH-KÜHNE), la reconstruction des universités (S. ZAUNER) ou la renaissance de la social-démocratie (E. WOLFRUM). On peut là encore regretter que les contributions restent dans un cadre géographique et chronologique un peu étroit, d'autant plus que l'ouvrage ne comporte pas de conclusion. C. RAUH-KÜHNE n'évoque ainsi que les mesures prises avant l'adoption au 1er juillet 1947 de la procédure des chambres d'épuration importée de la zone américaine, ce qui a cependant le mérite de relativiser la vision habituelle d'une 
dénazification très superficielle (la »Mitläuferfabrik« décrite par Lutz Niethammer). Des comparaisons plus fréquentes avec d'autres zones auraient cependant été bienvenues. Il est vrai que cette limite est largement liée au caractère pionnier des recherches présentées dans ce livre. Il faut souhaiter que d'autres études régionales aussi riches soient menées sur ce sujet en Allemagne.

Hervé JOLY 